\title{
EVALUATION OF ALLOCATION POLICIES ON HYBRID FIBER-COAX BROADBAND ACCESS NET- WORKS FOR CONTENTION-TYPE TRAFFIC
}

\author{
Cèsar Fernàndez* \\ Computer Science Department, University of Lleida (UdL) \\ Ap. de correus 471, 25080, Lleida, Spain \\ cesar@eup.udl.es
}

Sebastià Sallent

Telematics Engineering Department, Polytechnical University of Catalonia (UPC)

Gran Capità, s/n. M. C3, 08034, Barcelona, Spain

matssr@mat.upc.es

\begin{abstract}
Networks with centralized access mechanisms, e.g. HFC 802.14, are specified by a set of standards that in most cases does not cover internal operations of the central station, called Head-End Controller (HC) in HFC 802.14 networks. An example of this sort of operations could be the manner in which the HC allocates resources for contention-type traffic. In this paper we study two types of policies that the $\mathrm{HC}$ could use to allocate this kind of traffic. In both cases, we obtain computationally efficient analytical expressions of the mean Contention Resolution Period (CRP) using the methods described in [9] for a $Q$-ary resolution algorithm. Also we define two parameters of evaluation that in conjunction with the computations of the CRP could help to the HC to look for the best policy.
\end{abstract}

Keywords: Hybrid Fiber-Coax Networks, Medium Access Control Protocols, Contention Resolution Period Determination, Capacity Evaluation, Allocation Strategies.

\section{INTRODUCTION}

In recent years, it has been an obvious interest in offering higher capacity internet services to residential users. With this goal in mind, several alternatives have been heavily debated, from cooper loops as ADSL (Asymmetric 
Digital Subscriber Line) to FTTH (Fiber To The Home). One of these viable alternatives that might be taken into account is HFC (Hybrid Fiber Coax) due to several reasons, such as the high bandwidth of fiber optics and the large number of existing networks of coaxial cable where an important amount of bandwidth is not being used.

In this sense, the IEEE 802.14 working group has done a considerable work on the definition of the Physical and Medium Access Control layer protocols of a bi-directional Cable TV network based on HFC technology. The last public release of the standard, see [1], presents the architectural view of a 802.14 network, detailing protocols and services involved.

HFC networks are systems where the access procedures are centralized on a single station, called Head-End Controller (HC). As a first approach, the HC tasks could be divided into two categories; those related with the HC-stations communication procedures, covered by the standard, and those internal to the $\mathrm{HC}$ devoted to allocate resources in a efficient fashion, out of the standard scope. One of these internal tasks is debated in this paper.

The motivation of this paper is a lack of analytical performance literature that studies the allocation procedures of the HC. Much work developed in this area focuses on simulation results or in particular access mechanisms not closely related with the standard $[5 ; 3 ; 8 ; 10]$. In this sense, we propose and analyze two alternatives for the allocation of mini-slots accessed by the stations, in order to acquire HC granting to transmit without collision. In both cases, we obtain computationally efficient analytical expressions of the mean Contention Resolution Period (CRP) using the methods described in [9] for a $Q$-ary resolution algorithm. Also we define two parameters of evaluation, namely the capacity for contention-type traffic and the rate of global bandwidth devoted to carry out such a traffic. Both parameters in conjunction with the computations of the CRP could help to the $\mathrm{HC}$ to look for the best policy; i.e. the policy that maximizes $C$ under a given network load.

This paper is organized as follows: Section 2 introduces the basic aspects of the contention access mechanism for HFC 802.14 networks. Two possible strategies are defined. The first one, based on groups of mini-slots with fixed length and the second one with variable length groups. In Section 3 we obtain analytical results of the CRP for the fixed strategy. The same objectives are pursued in Section 4 for variable length groups. In Section 5 we define the evaluation parameters that will allow us to decide the better strategy to be applied by the HC under certain network scenarios. These parameters are numerically evaluated in Section 6 under Poisson traffic arrival. Finally, in Section 7 we conclude and discuss future lines of study. 

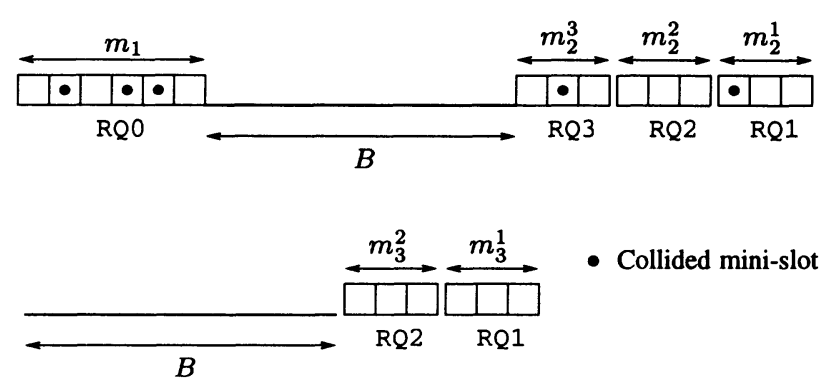

Figure 1 Schematic description of the HC contention mini-slot allocation policy.

\section{OVERVIEW OF THE CONTENTION ACCESS MECHANISM}

An HFC system can be represented as a group of stations linked to a HeadEnd Controller by means of two multiplexed frequency and unidirectional channels. The upstream channel, from stations to the HC, operating in the 5-50 $\mathrm{MHz}$ band, and the downstream channel, from the $\mathrm{HC}$ to stations, operating beyond $450 \mathrm{MHz}$ until $1 \mathrm{GHz}$. Every station communicates with each other through the $\mathrm{HC}$ accessing the upstream channel. The $\mathrm{HC}$ echoes upstream information to the downstream channel, also using this channel to broadcast upstream channel status information.

The main goal for HFC Medium Access Control designers has been how to efficiently use the upstream channel over a transmission speed range from 3 to $100 \mathrm{Mbps}$. In this sense, the time in the upstream channel is split up into data-slots and contention mini-slots. The size of data-slots could be enough to allocate an ATM cell, meanwhile the contention slots are shorter. Contention mini-slots are used by the stations to send allocation requests to the HC using a determined contention algorithm (Stabilized Aloha, n-ary tree, XDQRAP, ...) For every successful request, the HC informs about the status of reserved data-slots employing the downstream channel.

Our analysis scopes in the operations described in the standard drafts, where the slot structure is defined by the HC in a dynamical manner. Nevertheless, some simplifications have been done in order to make feasible an analytical approach. In this sense, the guidelines of the protocol operation are the following:

As showed in Figure 1, the HC allocates groups of request mini-slot according to a certain policy. The first group of a contention resolution period is called RQ0. All the subsequent groups of mini-slots will be devoted to resolve contentions produced in $\mathrm{RQO}$. $B$ mini-slots later, where $B$ has to be larger than the round trip delay, the $\mathrm{HC}$ allocates as many groups of mini-slots as 
those collided in RQ0. This is called a cluster. In Figure 1, the first cluster is composed of three groups of mini-slots called RQ3, RQ2 and RQ1. Each station involved in a request is informed by the downstream channel. If no collision exists, the $\mathrm{HC}$ tells to the station where to transmit its information. Otherwise, the HC informs to the station where is located the following group of request mini-slots devoted to resolve its contention. The request mini-slot is randomly chosen by the station. This operation is repeated until no collission is produced in any group. At this moment, the $\mathrm{HC}$ is able to allocate a new RQO.

Generally speaking, the size of $\mathrm{RQO}$ is $m_{1}$ mini-slots. This size can be modified by the $\mathrm{HC}$ at every round. The size of the groups belonging to cluster number $i$ is $m_{i}^{j}$, where the superscript $j$ denotes the group number $j$ inside the cluster. Our assumption along the paper is that $m_{i}^{j}=m_{i}$ for all $j$.

At this point, two policies can be identified according to the size of the groups of mini-slots belonging to a cluster. The first one is an allocation policy where the group's length is the same for every cluster. That is, $m_{i}=m$ for all $i$. The second one allows a reduction of the group's length depending on the cluster considered. This group's length reduction pursues a best utilization of the upstream channel bandwidth.

The first goal is to find a computationally efficient expression, that gives us the mean length of the contention resolution period (CRP). This period is taken to be the elapsed time between two successive RQ 0 clusters. Afterwards we define two kind of performance parameters in order to evaluate different policies.

\section{CLUSTERS WITH GROUPS OF FIXED LENGTH}

According to Figure 1, every group, independently of the cluster it belongs to, has the same length, say $m$ mini-slots. Define the mean CRP given that $N$ arrivals have occurred during last CRP as $Y_{N}^{m}$. In the same way that with others resolution mechanisms $[9 ; 7 ; 4], Y_{N}^{m}$ can be recursively expressed as

$$
Y_{N}^{m}=B+L_{N}^{m}=B+m+B P_{N}^{m}+\sum_{i_{1} \cdots i_{m}}^{N}\left(\begin{array}{c}
N \\
i_{1} \cdots i_{m}
\end{array}\right) \frac{1}{m^{i_{1}}} \cdots \frac{1}{m^{i_{m}}} \sum_{j=1}^{m} l_{i_{j}}^{m},
$$

where $P_{N}^{m}$ is the probability that a collission occurs in RQ 0 given that $N$ arrivals exist, namely

$$
\bar{P}_{N}^{m}=1-P_{N}^{m}=\prod_{i=1}^{N-1}\left(1-\frac{i}{m}\right)
$$


and $l_{i_{j}}^{m}$ can be expressed as

$$
l_{i_{j}}^{m}= \begin{cases}0 & i_{j}=0, i_{j}=1 \\ L_{i_{j}}^{m} & i_{j} \geq 2\end{cases}
$$

with $L_{0}^{m}=L_{1}^{m}=m$. In (3.1), $\sum_{i_{1} \cdots i_{m}}^{N}$ is the sum over all possible combinations of $i_{1} \cdots i_{m}$ such that equals $N$, and $\left(\begin{array}{c}N \\ i_{1} \cdots i_{m}\end{array}\right)$ is the multinomial coefficient. Manipulating (3.1) conveniently, as detailed in [9; 6], yields

$$
\begin{aligned}
L_{N}^{m}= & m+\sum_{i=0}^{\infty} m^{i}\left[m^{2}+B-B\left(1-m^{-i}\right)^{N}-m^{2}\left(1-m^{-i-1}\right)^{N}\right. \\
& \left.-B \sum_{j=2}^{\min (N, m)}\left(\begin{array}{c}
N \\
j
\end{array}\right) \bar{P}_{j}^{m}\left(1-m^{-i}\right)^{N-j} m^{-j i}\right] \\
& -\sum_{i=0}^{\infty}\left[B N\left(1-m^{-i}\right)^{N-1}+m N\left(1-m^{-i-1}\right)^{N-1}\right] .
\end{aligned}
$$

\section{CLUSTERS WITH GROUPS OF VARIABLE LENGTH}

One could think, in contrast to the previous policy, an allocation policy that periodically reduces the length of the groups of mini-slots at every cluster delivered to the network. As we demonstrate later, a first approach could be to destinate the longest group ( $\mathrm{RQ} 0$ ) to start the first collission resolution, where more arrivals remain to be solved, and to decrease progressively the length of the groups of every cluster until reaching a minimum.

Here we consider a policy based on the length reduction by a constant factor that divides the length, say $r$. In an extended version of this paper ([6]), we study a more general method, where the length of the groups does not necessarily obeys a determined rule. In both cases, we assume the same length in any group inside a cluster, that is, $m_{i}^{j}=m_{i}$ for all $j$, according to the notation introduced in Figure 1.

Let $m_{i}$ be the length of any group of mini-slots in cluster number $i$. Take cluster number 1 as that which contains $\mathrm{RQO}$. The length for groups inside the next cluster is computed according a transformation $\sigma()$ as $m_{i+1}=\sigma_{r, m_{\infty}}\left(m_{i}\right)$, where

$$
\sigma_{r, m_{\infty}}(x)= \begin{cases}\left\lfloor\frac{x}{r}\right\rfloor+1 & \text { if }\left\lfloor\frac{x}{r}\right\rfloor+1 \geq m_{\infty} \\ m_{\infty} & \text { otherwise }\end{cases}
$$

and where $\lfloor y\rfloor$ denotes the integer part of $y, r \geq 2$ is an integer constant and $m_{\infty} \leq m_{1}$. The fact of adding 1 is not relevant, but it is maintained because formulation of $\sigma()$ for $r=2$ was more compact on preliminary analysis. 
In both strategies, constant factor and generic reduction, we will obtain expressions of $Y_{N}^{m_{1}}$ for $m_{\infty}=2$. Other cases are left to further studies.

As done with the fixed length strategy, recurrence in $N$ must be avoided if we intend to perform calculations for large $N$. So, we define a two variant generating function, indexing $N$ and $m$. Resolution is detailed in [6]. Finally, we obtain

$$
L_{N}^{m_{1}}=m+\sum_{k=2}^{N} \frac{N !}{(N-k) !} H_{k}^{m_{1}},
$$

where

$$
\begin{aligned}
H_{k}^{m_{1}}= & \frac{1}{m_{1} k-1} H_{k}^{\left\lfloor m_{1} / r\right\rfloor+1}+B \frac{(-1)^{k}}{k !}(k-1) \\
& +\left(\left\lfloor m_{1} / r\right\rfloor+1\right) m_{1} \frac{\left(-1 / m_{1}\right)^{k}}{k !}(k-1) \\
& -B \sum_{i=2}^{\min (k, m)} \bar{P}_{i}^{m_{1}} \frac{1}{i !} \frac{(-1)^{k-i}}{(k-i) !} .
\end{aligned}
$$

\section{EVALUATION PARAMETERS}

Once the mean CRP can be calculated numerically, we are able to obtain two important measures that describe the performance of the access mechanism when contention type traffic is considered.

The first parameter, subsequently called $R$, is the fraction of the total amount of available bandwidth dedicated to contention traffic. Namely, how many minislots are dedicated to solve contentions in contrast to those used to transfer information on them. $R$ is a percentage.

The second parameter, say $C$, is the capacity of the access mechanism for contention type traffic. $C$ is a throughput and is calculated in number of mini-slots by time of mini-slot.

In any case, both parameters depend on the mean CRP given than $N$ arrivals are produced on previous CRP, $Y_{N}$, and on the probability that $N$ arrivals were produced given a predefined tax $\lambda$. We call this probability $P_{N}(\lambda)$.

Let $Y_{N}(B)$ be the mean CRP for a particular strategy given that $N$ arrivals must be allocated, considering that the $\mathrm{HC}$ has decided an inter-cluster gap of $B$ mini-slots. It is clear that

$$
R=\frac{\sum_{i=0}^{\infty} P_{i}(\lambda) Y_{i}(0)}{\sum_{i=0}^{\infty} P_{i}(\lambda) Y_{i}(B)}
$$

On the other hand, $C$ can be calculated according to the definition of capacity of a resolution contention algorithm introduced by [7]

$$
C=\sup _{\lambda} \frac{\lambda}{\sum_{i=0}^{\infty} P_{i}(\lambda) Y_{i}(B)} .
$$


Clearly, the objective of our computations is to look for the best strategy used by the $\mathrm{HC}$ in order to allocate the contention clusters.

\section{NUMERICAL RESULTS}

This section shows several calculations performed under different network scenarios. The analyzed strategies have been basically two, clusters with groups of fixed length and clusters with groups of variable length with a constant factor reduction strategy. In the later case, many factors of reduction have been explored.

First of all, let us study the performance of the access mechanism when immediate feedback is considered $(B=0)$, in order to compare its capacity with others appeared in the literature. Afterwards, we show the throughput and the occupation rate for different values of $B$, helping this results the $\mathrm{HC}$ to look for the best policy. It is important to note that all the results are obtained considering arrival inputs as Poisson distributed, as well as those reported from alternate mechanisms.

The best capacity of HFC 802.14 algorithm for $B=0$ when a fixed length policy is used is achieved for a group length $m=2$. Its value is $C=0.429512$. Variable length strategies do not introduce any improvement in $C$ for $B=0$.

Several proposals have been submitted to the committee during the elaboration of the 802.14 standard. These proposals include many contention access protocols, most of them studied some years ago when a lot of work was developed related to the evaluation performance of slotted contention networks. Some of these protocols and its corresponding capacities are p-persistent algorithms [2] (0.3679), tree-based ternary with free and blocked access [9] $(0.4016,0.3662)$, and first-come first-serve splitting ( $f c f s)$ [2] (0.4878).

Let us now inspect the system performance for $B \neq 0$. At this moment, intensive calculations have been done for two values of $B, B=20$ and $B=100$. They corresponds to a network of 9,6 and $48 \mathrm{Km}$ length respectively, considering $10 \mathrm{Mbps}$ in the upstream channel and a mini-slot length of 6 bytes.

Figure 2 (left) shows the capacity versus contention rate bandwidth for a network of $B=20$. As an example, fixed and variable constant rate $(r=2$ and $r=5$ ) strategies are plotted. Six points for fixed strategy are shown, corresponding to a range from $m=3$ until $m=8$. As can be seen, increasing $m$ implies a better capacity but a larger bandwidth use, from $20 \%$ until more than $50 \%$ for $m=8$. Also it is noted that a variable strategy with $r=2$ outperforms in all cases than the fixed strategy. Moreover, the variable strategy with $r=5$ outperforms $r=2$, more clearly for large values of $R$. Note that any point of a plot corresponds to a certain value of $m$. 

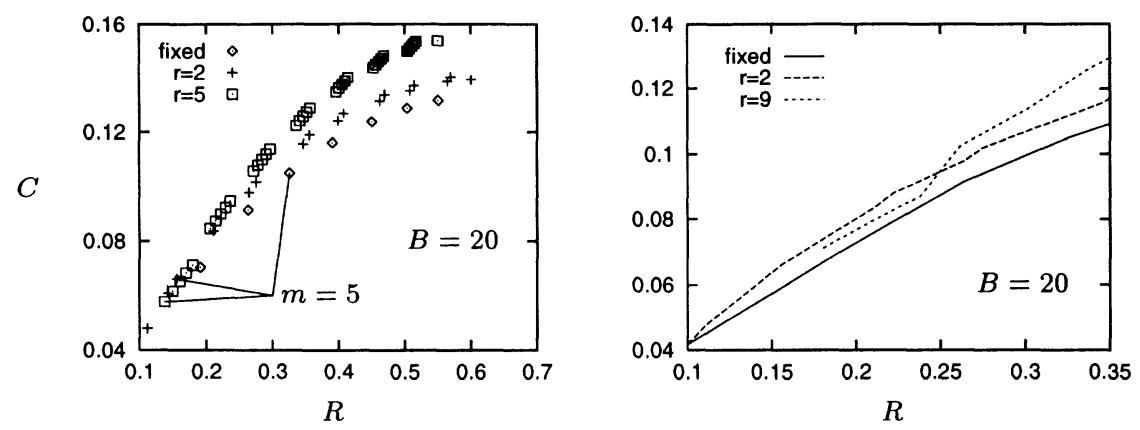

Figure $2 C$ versus $R$ performance for $B=20$

\begin{tabular}{llll}
\hline \multicolumn{2}{c}{$B=20$} & \multicolumn{2}{c}{$B=100$} \\
\hline$R$ Range & Strategy & \multicolumn{1}{c}{$R$ Range } & Strategy \\
\hline$[0,0.16]$ & $r=2$ & {$[0,0.035]$} & $r=2$ \\
{$[0.16,0.19]$} & $r=3$ & {$[0.035,0.055]$} & $r=3$ \\
{$[0.19,0.21]$} & $r=4$ & {$[0.055,0.105]$} & $r=4$ \\
{$[0.21,0.3]$} & $r=5$ & {$[0.105,0.125]$} & $r=5$ \\
{$[0.3,0.35]$} & $r=7$ & {$[0.125,0.17]$} & $r=7$ \\
{$[0.35,0.4]$} & $r=9$ & {$[0.17,0.19]$} & $r=9$ \\
{$[0.4,0.6]$} & $r=13$ & {$[0.19,0.22]$} & $r=11,13$ \\
\hline
\end{tabular}

Table 1.1 Optimum strategy depending on $R$

But the question is: Which is the best choice of $r$ for a variable constant rate strategy? The answer depends on the range of $R$ considered. Figure 2 (right) plots the throughput of a system with $B=20$ for contention traffic rates from $10 \%$ until $35 \%$. At a first glance, this plot shows how the best strategy depends on $R$. More accurately, Table 1.1 lists the optimum strategy for a disjoint set of ranges of $R$, for $B=20$ and $B=100$ (only odd values of $r$ beyond 5 have been used).

In this way, the $\mathrm{HC}$ is able to choose the optimum strategy calculating $C$ versus $R$ performance measurements for several values of $B$, namely, such strategy that maximizes throughput under a certain available bandwidth. Furthermore, if not enough bandwidth is available, the $\mathrm{HC}$ could increase $B$ in order to find the first value of $B$ that allows contention traffic allocation using $r=2$. 


\section{CONCLUSIONS AND FUTURE WORK}

In this paper we have studied two allocation policies used by the $\mathrm{HC}$ for the access of contention-type traffic. We have generalized the results of [9] and we have applied them to an allocation strategy where the splitting factor of the algorithm is variable and where delay is considered.

The obtained CRP expressions allow the computation of CRP for large values of $N$ in short time. These expressions have been validated comparing the results against the recursive expressions for small values of $N$. Once calculated the $\mathrm{CRP}$, this parameter is used to obtain the capacity $(C)$ versus contention traffic bandwidth requirement $(R)$, obtaining the best policy depending on $R$. As shown in Section 6, constant factor reduction strategies clearly outperforms the fixed policy when Poisson traffic is assumed, so look up tables such as Table 1.1 could help the HC to decide which is the best strategy under certain resource availability conditions for contention traffic.

Nevertheless, more work has to be done in this sense. First, expressions for $Y_{N}^{m_{1}}$ have to be generalized for $m_{\infty}>2$. Second, some generic reduction strategies have to be tested in order to compare their performance against constant rate reduction. An example of generic reduction strategies could be a sequence $m_{i}$ defined as a Fibonacci series. And third, other type of arrival processes could introduce variations in the look up tables of $C$ versus $R$.

\section{References}

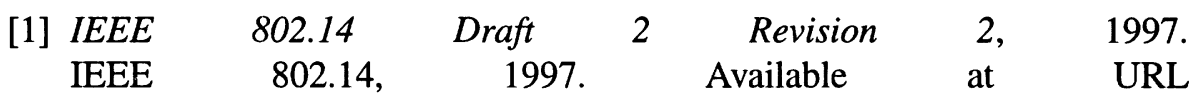
http : / / texcat.com/users/chicago/draft2-rev2 .pdf.

[2] Dimitri P. Bertsekas and Robert Gallager. Data Networks. Prentice Hall, 1992.

[3] Chatschik Bisdikian, Bill McNeil, Rob Norman and Ray Zeisz. MLAP: A MAC Level Access Protocol for the HFC 802.14 network. IEEE Communications Magazine, 34(3):114-121, March 1996.

[4] John I. Capetanakis. Tree algorithms for packet broadcast channels. IEEE Transactions on Information Theory, 25(5):505-515, May 1979.

[5] James E. Dail, Miguel A. Dajer, Chia-Chang Li, Peter D. Nagill, Curtis A. Siller, Kotikalapudi Sriram and Norman A. Whitaker. Adaptative digital access protocol: A MAC protocol for multiservice broadband access networks. IEEE Communications magazine, 34(3):104-113, March 1996.

[6] Cèsar Fernàndez and Sebastià Sallent. Evaluation of allocation policies on hybrid fiber-coax broadband access networks for contention-type traffic (extended version). Technical Report DIEI-99-RT-2, Departament d'Informàtica i Enginyeria Industrial. Universitat de Lleida, April 1999. 
[7] Huang Jian-Cheng and Toby Berger. Delay analysis of interval-searching contention resolution algorithms. IEEE Transactions on Information Theory, 31(2):264-273, March 1985.

[8] Ying-Dar Lin, Chia-Jen Wu and Wei-Ming Yin. PCUP: Pipelined Cyclic Upstream Protocol over Hybrid Fiber Coax. IEEE Network, 11(1):24-34, January/February 1997.

[9] Peter Mathys and Philippe Flajolet. Q-ary collision resolution algorithms in random-access systems with free or blocked channel access. IEEE Transactions on Information Theory, 31(2):217-243, February 1985.

[10] Dolors Sala and John O. Limb. Comparison of contention resolution algorithms for a cable modem MAC protocol. In International Zurich Seminar on Broadband Communications, February 1998. 